\title{
Novel insights into capillary vessel basement membrane damage by snake venom hemorrhagic metalloproteinases: A biochemical and immunohistochemical study
}

\author{
Teresa Escalante a , John Shannon ${ }^{\mathrm{b}}$, Ana M. Moura-da-Silva ${ }^{\mathrm{c}}$, \\ José María Gutiérrez ${ }^{\mathrm{a}}$, Jay W. Fox ${ }^{\mathrm{b}, *}$ \\ a Instituto Clodomiro Picado, Facultad de Microbiología, Universidad de Costa Rica, San José, Costa Rica \\ ${ }^{\mathrm{b}}$ Department of Microbiology, Health Sciences Center, University of Virginia, Jordan Hall, P. O. Box 800734, Charlottesville, VA 22908-0734, USA \\ ${ }^{\mathrm{c}}$ Laboratorio de Imunopatologia, Instituto Butantan, Sao Paulo, Brazil
}

Received 9 August 2006, and in revised form 15 September 2006

Available online 6 October 2006

\begin{abstract}
The hemorrhagic activity characteristic of viperid snake envenomations is due to the action of venom metalloproteinases (SVMPs) on the capillary vessel basement membrane (BM). This study compared the action of two SVMPs on BM in vitro (degradation of Matrigel) and in vivo (immunohistochemical assessment of BM markers in mouse gastrocnemius muscle). SVMPs BaP1 (belonging to the P-I class) and jararhagin (of the P-III class) had a similar proteolytic activity on azocasein and degraded Matrigel with a slightly different cleavage pattern, since $\mathrm{BaP} 1$ exerted a limited proteolysis of both laminin and nidogen, whereas jararhagin predominantly degraded nidogen. In contrast with this pattern of limited proteolysis of BM proteins observed in vitro, immunohistochemical analysis of laminin, nidogen and type IV collagen, as well as of the endothelial cell marker VEGFR-2, in the hemorrhagic areas in the muscle, revealed a pronounced reduction in the immunostaining of these three BM components, associated with a loss of the endothelial cell marker. BM of muscle fibers was affected to a lesser extent. In conclusion, in vitro results demonstrated that SVMPs induce a pattern of limited proteolysis on BM components. The drastic loss of these antigens in affected capillaries in vivo is likely to depend on the combination of limited proteolysis of $\mathrm{BM}$ and the action of hemodynamic biophysical forces, previously shown to play a role in SVMP-induced capillary damage, which may cause a mechanical disruption of BM structure.
\end{abstract}

(C) 2006 Elsevier Inc. All rights reserved.

Keywords: Snake venom; Metalloproteinase; Basement membrane; Endothelial cells; Hemorrhage; Extracellular matrix

Envenomations by snakes of the family Viperidae induce multiple deleterious effects, including local and systemic bleeding [1,2]. Hemorrhagic toxins of viperid venoms are zinc-dependent metalloproteinases which, together with the ADAMS ( $A$ Disintegrin $A$ nd $M$ etalloproteinase) ${ }^{1}$ comprise the M12 reprolysin family of metalloproteinases [3]. Depending on their domain constitution, snake venom

\footnotetext{
* Corresponding author. Fax: +1 8049822514.

E-mail address: jwf8x@virginia.edu (J.W.Fox).

${ }^{1}$ Abbrevations used: ADAMS, A Dsintegrin And Metalloproteinase; SVMPs, snake venom metalloproteinases; VEGFR-2, vascular endothelial growth factor receptor 2, BM; basement membrane.
}

metalloproteinases (SVMPs) are classified within four main classes; the mature proteins of class P-I are composed of the metalloproteinase domain, having a characteristic zincbinding motif [4]. The other classes (P-II to P-IV) present, besides this catalytic domain, additional domains such as disintegrin (or disintegrin-like), cysteine-rich and C-type lectin-like domains, with additional variations within each class [3]. Despite sharing a very similar zinc-binding motif, not all SVMPs are able to induce hemorrhage; as a general rule, P-III SVMPs, comprised of metalloproteinase, disintegrin-like and cysteine-rich domains, are more potent hemorrhagic components than P-I SVMPs [5]. 
The mechanism of action of hemorrhage SVMPs has been investigated using various methodologies (for reviews see References 6,7), although the precise molecular and cellular events associated with microvessel disruption remain largely unknown. Enzymatic hydrolysis of basement membrane (BM) components has been proposed as a key event in the onset of capillary vessel disruption [5,8]. In vitro, SVMPs are able to digest the BM preparation Matrigel [9], as well as purified BM components [9-14]. Most of these analyses have been performed by observing the patterns of hydrolysis in SDS-PAGE. However, the characterization of the cleavage patterns on BM components of hemorrhagic and non-hemorrhagic SVMPs, as well as of SVMPs of different classes, has not been performed in detail, with the exception of atrolysin $e$, a P-I SVMP from the venom of the rattlesnake Crotalus atrox $[5,11,12,15]$. BM is a complex extracellular matrix structure comprising several major components, i.e. laminin, type IV collagen, nidogen/ entactin and the proteoglycan perlecan, in addition to a number of minor components [16]. BM is a supramolecular lattice which, among other functions, provides mechanical stability to the capillary vessel structure [16]. Hence, SVMPs that have different degradation patterns on BM proteins are likely to affect the mechanical stability of this network to different extents, depending on the effect that particular cleavage patterns exert in the mechanical properties of this lattice. Therefore, the characterization of cleavage patterns in BM by different SVMPs represents a relevant task. Moreover, whether SVMPs are also able to degrade BM components in vivo, and the extent of such degradation, are issues that have not been thoroughly investigated.

The presence of disintegrin-like and cysteine-rich domains in P-III SVMPs has been postulated as playing a critical role in their potent hemorrhagic activity [5]. Such high hemorrhagic potential may be due, at least partially, to the targeting of SVMPs to relevant locations in the capillary wall, such as endothelial cell integrins [17], or through the binding of exosites located in such additional domains to specific sequences in BM and other extracellular matrix components $[18,19]$, thus contributing to a more localized, specific and hence efficient BM degradation. Thus, the analysis of the degradation patterns of BM substrates in vitro and in vivo constitutes relevant issues to deepen our understanding of the mechanism of action of hemorrhagic SVMPs.

In this work, a comparison has been performed between two well-characterized hemorrhagic SVMPs: BaP1, a P-I enzyme from Bothrops asper venom which has been considered as weakly hemorrhagic [20], and jararhagin, a P-III enzyme from Bothrops jararaca venom which has a more potent hemorrhagic activity [21]. The goals of this study were to compare: (a) the turnover rate of these SVMPs on azocasein; (b) the degradation patterns on BM components incubated with these SVMPs in vitro; and (c) the ability of these SVMPs to alter the immunostaining of BM components of muscle capillaries in vivo.

\section{Materials and methods}

\section{Metalloproteinases}

$\mathrm{BaP} 1$, a $22 \mathrm{kDa}$ class P-I SVMP, was isolated from the venom of $B$. asper [20,22]. Jararhagin, a $52 \mathrm{kDa}$ class P-III SVMP, was isolated from the venom of $B$. jararaca [21].

\section{Degradation of azocasein}

Proteolytic activity of $\mathrm{BaP1}$ and jararhagin was tested on azocasein (Sigma-Aldrich, St. Louis, MO) according to Wang et al. [23], with modifications. Briefly, various concentrations of each SVMP, dissolved in $20 \mu \mathrm{L}$ of $25 \mathrm{mM}$ Tris, $150 \mathrm{mM} \mathrm{NaCl}, 5 \mathrm{mM} \mathrm{CaCl}_{2}, \mathrm{pH} 7.4$, were incubated with a $5 \mathrm{mg} / \mathrm{mL}$ solution of azocasein in the same buffer. After an incubation of $90 \mathrm{~min}$ at $37^{\circ} \mathrm{C}$, the reaction was stopped by the addition of $200 \mu \mathrm{L}$ of $5 \%$ trichloroacetic acid. Samples were then centrifuged at $2000 \mathrm{rpm}$. Supernatant $(150 \mu \mathrm{L})$ was diluted with $150 \mu \mathrm{L} 0.5 \mathrm{M} \mathrm{NaOH}$, and the absorbances were measured at $450 \mathrm{~nm}$. One unit of proteolytic activity corresponds to the amount of enzyme that induces a change in absorbance of 0.2 .

\section{Degradation of Matrigel}

Hydrolysis of Matrigel TM (BD Biosciences Discovery Labware, Bedford, MA) was assessed by incubating this BM preparation with either $\mathrm{BaP} 1$ or jararhagin at an enzyme:substrate ratio (w:w) of 1:50. Incubations of SVMPs and Matrigel were performed for $15 \mathrm{~min}, 1$ and $3 \mathrm{~h}$ at $37^{\circ} \mathrm{C}$. Reactions were stopped by the addition of one volume of $2 \times$ SDS-PAGE buffer (reducing conditions) [24]. A sample of Matrigel, incubated without SVMPs under otherwise identical conditions and at the same time intervals, was used as control. Electrophoresis was performed using a $4-15 \%$ Tris- $\mathrm{HCl}$ polyacrylamide gradient gel (Bio Rad Labs, Hercules, CA), and the bands were visualized with Coomassie Brilliant Blue staining. For the identification of cleavage sites of Matrigel proteins, incubations with either $\mathrm{BaP} 1$ or jararhagin were carried out as described for $1 \mathrm{~h}$ at $37^{\circ} \mathrm{C}$. After SDS-PAGE in a $4-15 \%$ gradient gel, the samples were transferred to immobilon strips and the bands visualized with Coomassie Brilliant Blue

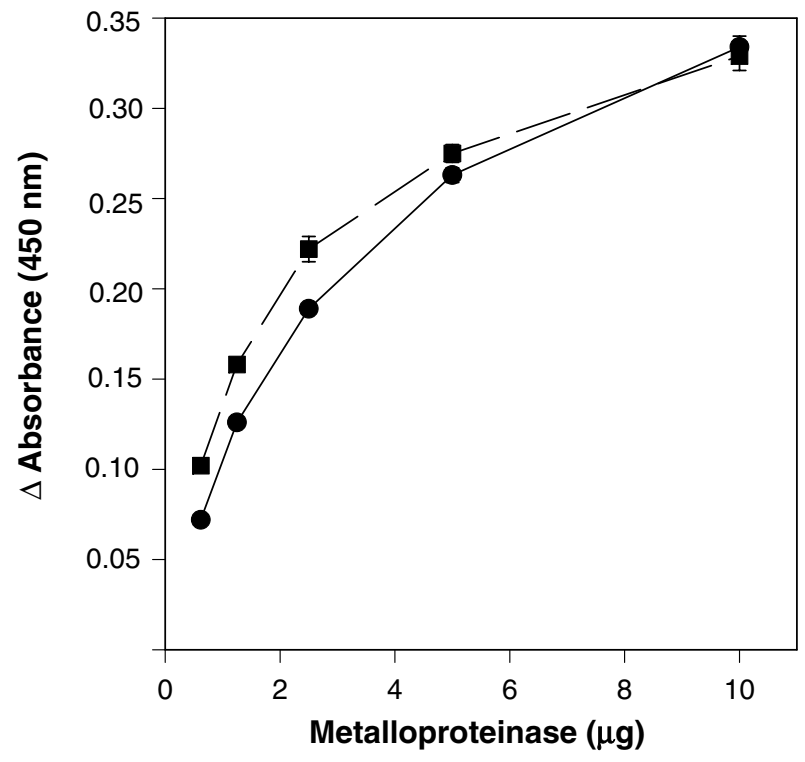

Fig. 1. Hydrolytic activity of BaP1 (ם) and jararhagin () on azocasein. Various amounts of each enzyme were incubated with azocasein for 90 min at $37^{\circ} \mathrm{C}$, as described in Materials and Methods. Reaction was stopped by the addition of $5 \%$ trichloroacetic acid, and the absorbances of the supernatants at $450 \mathrm{~nm}$ were recorded. Controls of azocasein without enzyme were run in parallel and their absorbance was subtracted from the sample values. Results are presented as mean $\pm \operatorname{SD}(n=3)$. 
staining. N-terminal sequence of degradation bands was determined in an Applied Biosystems Procise 494 protein sequencer instrument (following the manufacturer's recommendations)

\section{Immunocytochemistry}

Groups of CD-1 mice (18-20g) were injected intramuscularly, in the right gastrocnemius, with either saline solution $(100 \mu \mathrm{L}$ control), BaP1 $(30 \mu \mathrm{g} / 100 \mu \mathrm{L}$ or $2 \mu \mathrm{g} / 100 \mu \mathrm{L})$, or jararhagin $(5 \mu \mathrm{g} / 100 \mu \mathrm{L})$. Thirty micrograms of $\mathrm{BaP} 1$ and $5 \mu \mathrm{g}$ of jararhagin induced prominent hemorrhage in the injected muscle; $2 \mu \mathrm{g}$ BaPl did not induce hemorrhage and was selected in order to compare the activity of $\mathrm{BaP} 1$ and jararhagin at the same molar basis. At $15 \mathrm{~min}$ and $1 \mathrm{~h}$ after injection, mice were sacrificed with an overdose of $\mathrm{CO}_{2}$ and the injected muscle was dissected out and immediately placed in cold isopentane and frozen in liquid nitrogen. Sections of $8 \mu \mathrm{m}$ were cut in a cryotome (Leica CM 1850, Nussloch, Germany) and fixed in $100 \%$ ethanol. Sections were incubated with either antimouse laminin polyclonal antibody (Research Diagnostics Inc., Concord,
MA), at a 1:3000 dilution, anti-mouse type IV collagen polyclonal antibody (Research Diagnostics Inc.,) at a 1:3000 dilution, anti-mouse nidogen polyclonal antibody (Chemicon) at a 1:8000 dilution, or anti-mouse vascular endothelial growth factor receptor 2 (VEGFR-2) monoclonal antibody (BD Biosciences Pharmingen, San Diego, CA) at a 1:20 dilution. The reaction was detected with the tyramide signal amplification kit (Perkin Elmer, Boston, MA) using biotinylated secondary antibodies. Visualization of VEGFR-2 was performed with streptavidin-fluorescein (DakoCytomation, Glostrup, Denmark) and BM antigens with streptavidin-Cy3 (Zymed Laboratories Inc., South San Francisco, CA). Experiments involving mice were approved by the Committee for the Use and Care of Laboratory Animals (CICUA) of the University of Costa Rica.

In order to have a quantitative assessment of capillary BM alterations, sections from damaged areas were analyzed using the $20 \times$ objective lens, and the total number of capillaries and muscle cells were counted, in order to obtain the capillary:muscle cell ratio. This was possible since muscle fibers can be identified on the basis of immunostaining of their BM, which is less severely affected than BM of capillaries and,

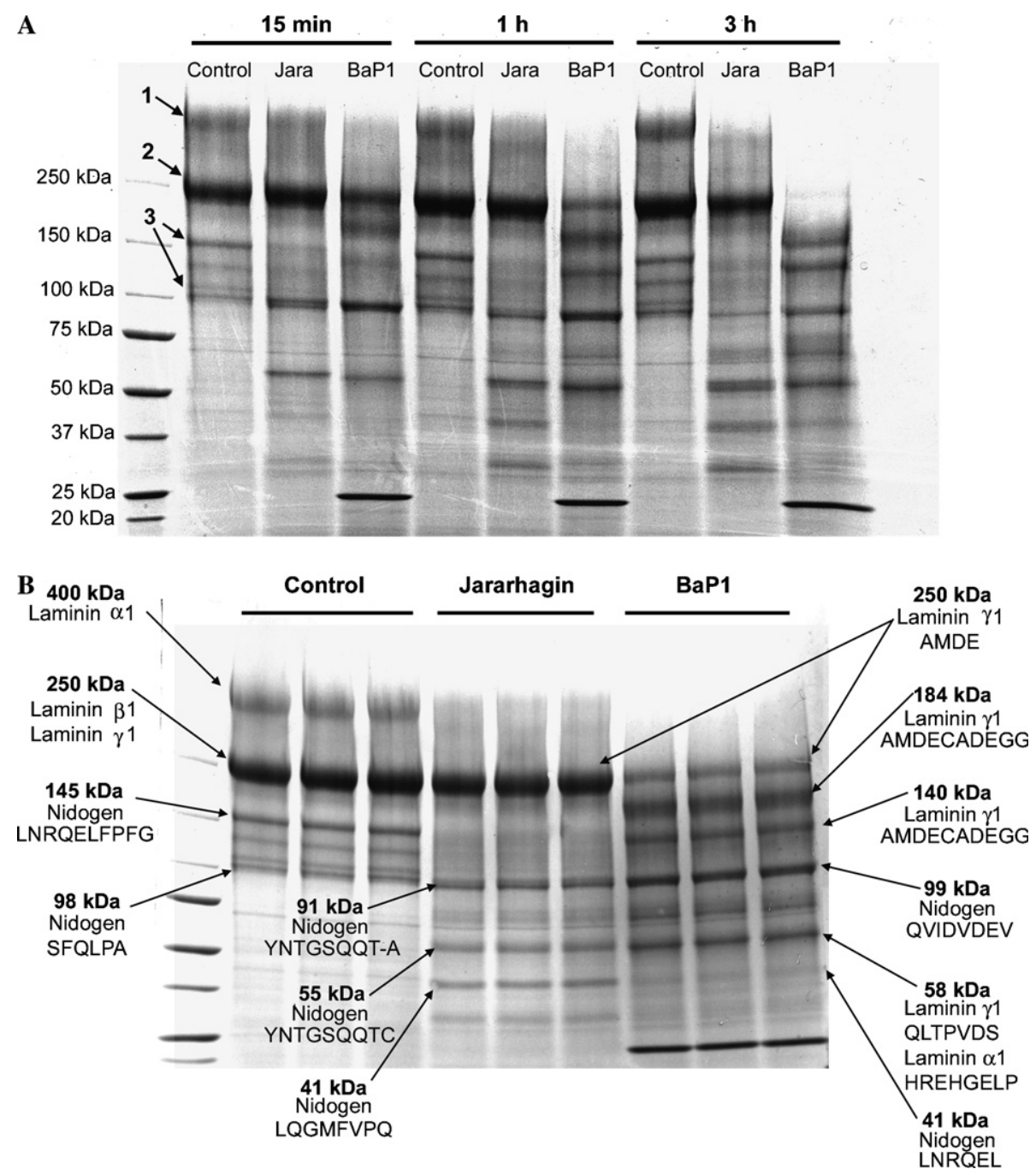

Fig. 2. Hydrolysis of Matrigel proteins by BaP1 and jararhagin. (A) Matrigel was incubated with each enzyme at a 1:50 (w:w) enzyme:substrate ratio for various time intervals. A control of Matrigel preparation incubated without enzymes was included at each time interval. Reactions were stopped by the addition of SDS-PAGE reducing sample buffer, and mixtures separated by SDS-PAGE, run under reducing conditions, using a 4-15\% polyacrylamide gradient gel. Molecular mass markers were included (left lane). Gels were stained with Coomassie Brilliant Blue. In the controls, the most abundant Matrigel bands correspond to (1) Laminin $\alpha-1$ chain, (2) laminin $\beta$ and $\gamma$ chains, (3) Nidogen chains. There is degradation of Matrigel components by the two SVMPs as early as $15 \mathrm{~min}$ of incubation. (B) Identification of the cleavage sites of Matrigel components (laminin and nidogen chains) upon $1 \mathrm{~h}$ of incubation with $\mathrm{BaP} 1$ or jararhagin at a 1:50 (w:w) enzyme:substrate ratio. The molecular mass of degradation fragments is indicated, as well as their $\mathrm{N}$-terminal sequence. Samples were analyzed in triplicates. BaP1 generated cleavage fragments of both laminin and nidogen chains, whereas jararhagin generated mostly fragments derived from nidogen. 
therefore, allow the identification of all muscle fibers in a given tissue section, even in damaged areas. The capillary:muscle cell ratio is commonly used in the study of microvascular alterations in skeletal muscle [25]. For these analyses, three to four sections were analyzed in each sample for each BM antigen.

\section{Statistical analysis}

To assess the statistical significance of the differences in the mean values of capillary:muscle cell ratios, an analysis of variance was performed, followed by a Tukey-Kramer test to compare pairs of means.

\section{Results}

\section{Degradation of azocasein and matrigel}

BaP1 and jararhagin showed similar dose-response curves of proteolytic activity when tested on azocasein (Fig. 1). Activity was 500 and $400 \mathrm{U} / \mathrm{mg}$ for BaP1 and jararhagin, respectively. However, in terms of molar basis, jararhagin showed a higher activity than BaP1 (11 vs $21 \mathrm{U} /$ nmol), owing to the different molecular mass of these SVMPs. BaP1 and jararhagin were also tested for their ability to hydrolyze Matrigel. After 15 min of incubation, $\mathrm{BaP} 1$ cleaved nidogen as well as laminin $\alpha$ and $\gamma$ chains, whereas jararhagin predominantly degraded nidogen (Fig. 2A). At $1 \mathrm{~h}$ incubation with jararhagin, a reduction in the intensity of laminin $\alpha$ chain was observed, although $\beta$ and $\gamma$ chains were apparently not degraded. Samples collected after $3 \mathrm{~h}$ incubation show a complete degradation of the three bands of laminin by $\mathrm{BaP} 1$, whereas jararhagin had completely degraded laminin $\alpha$ chain; in addition, the band corresponding to $\beta$ and $\gamma$ laminin chains was reduced in intensity (Fig. 2A). The $\mathrm{N}$-terminal sequence of the main degradation fragments was determined after $1 \mathrm{~h}$ incubation of Matrigel with $\mathrm{BaP1}$ or jararhagin. As shown in Fig. 2B, $\mathrm{BaP} 1$ generated fragments from the laminin $\alpha$ and $\gamma$ chains, as well as of nidogen, whereas jararhagin predominantly generated fragments derived from nidogen.

\section{Immunocytochemistry}

When sections of muscle tissue from mice injected with saline solution were immunostained with anti-VEGFR-2, a marker of endothelial cells, a typical distribution of capillary blood vessels was evidenced (Fig. 3A). There were several capillaries surrounding each muscle fiber in a
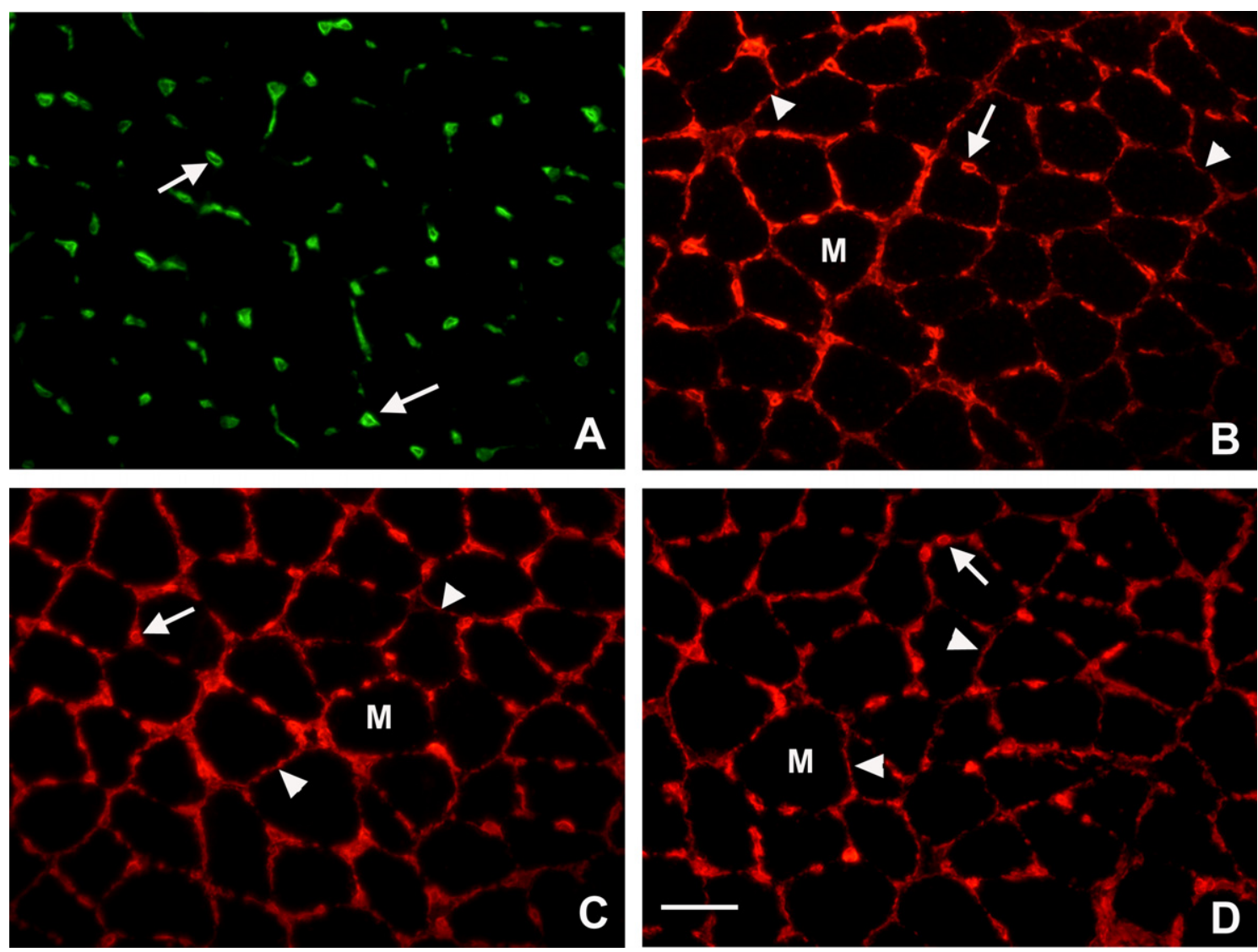

Fig. 3. Immunohistochemical staining for endothelial and BM markers in serial cryosections of skeletal muscle tissue (gastrocnemius) of control mice 15 min after injection of saline solution. The following primary antibodies were used: (A) anti-mouse vascular endothelial growth factor receptor 2 (VEGFR-2), (B) anti-mouse laminin, (C) anti-mouse nidogen, (D) anti-mouse type IV collagen. Reactions were detected with a tyramide signal amplification kit using biotinylated secondary antibodies. Visualization of VEGFR-2 was performed with streptavidin-fluorescein, and BM antigens with streptavidin-Cy3. Capillaries are clearly identified in the endomysium (arrow), and a continuous BM (arrow head) is observed in the periphery of skeletal muscle fibers (M). Bar represents $40 \mu \mathrm{m}$. 
characteristic arrangement for the microvasculature of this tissue [26]. A similar pattern was observed when sections were incubated with anti-CD 31, another marker of endothelial cells (not shown). Immunostaining of sections from control mice with antibodies anti-type IV collagen, laminin and nidogen revealed a staining pattern that was located in capillary vessels and in the periphery of muscle fibers (Fig. 3B, C and D), corresponding to BMs of these two structures.

Tissue injected with $30 \mu \mathrm{g}$ BaP1 showed extensive areas of hemorrhage, evidenced by the presence of extravasated erythrocytes located in the perimysium and endomysium in samples collected at $15 \mathrm{~min}$ and $1 \mathrm{~h}$ after toxin injection, in agreement with previous studies [14]. Staining for endothelial cell markers was drastically reduced in the majority of these hemorrhagic areas (Fig. 4A, C and E). In addition, the patterns of immunostaining for the BM components laminin (Fig. 4B), nidogen (Fig. 4D) and type IV collagen (Fig. 4F) were altered in the capillary vessels of these areas, with an evident reduction in the number of capillaries stained for these antigens, as corroborated quantitatively when the capillary:muscle cell ratio was determined in these
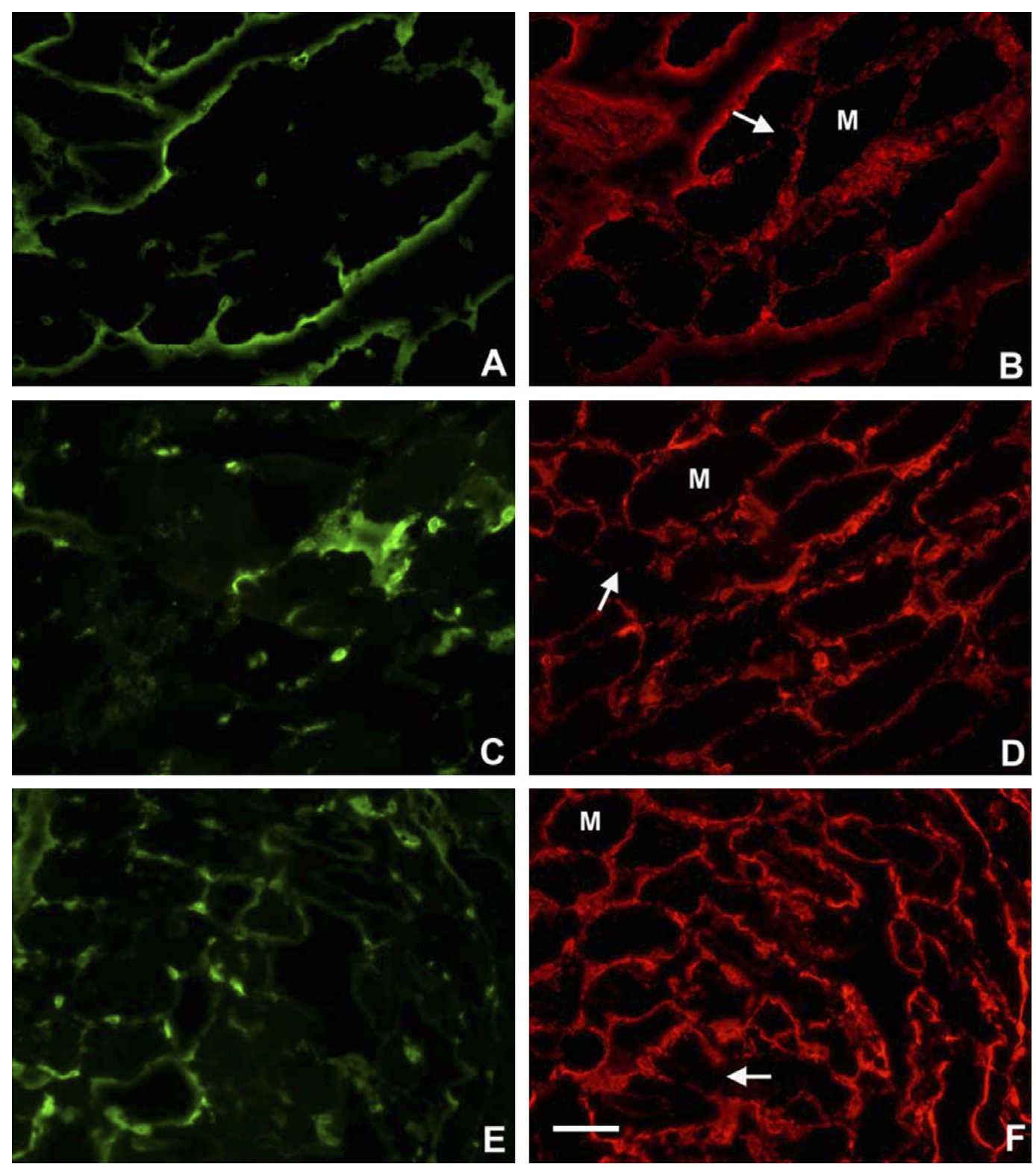

Fig. 4. Immunohistochemical staining for endothelial and BM markers in serial cryosections of skeletal muscle tissue (gastrocnemius) of mice 15 min after i.m. injection of $30 \mu \mathrm{g} \mathrm{BaP1}$. Sections correspond to areas showing evidences of tissue damage, i.e. hemorrhage. The following primary antibodies were used: (A, C, E) anti-mouse vascular endothelial growth factor receptor 2 (VEGFR-2), (B) anti-mouse laminin, (D) anti-mouse nidogen, (F) anti-mouse type IV collagen. Sections A and B, C and D, and E and F correspond to serial sections from the same tissue sample. Immunostaining was performed as described in the legend of Fig. 3. There is a drastic reduction in the immunostaining of VEGFR-2 and BM markers in capillaries. There is a diffuse staining in (A) and (C) that does not correspond to endothelial cells. BMs of skeletal muscle cells show some alterations, i.e. reduction in the intensity of immunostaining and interruption in their continuity (arrow), although such alterations were less pronounced that those observed in capillaries. Bar represents $40 \mu \mathrm{m}$. 


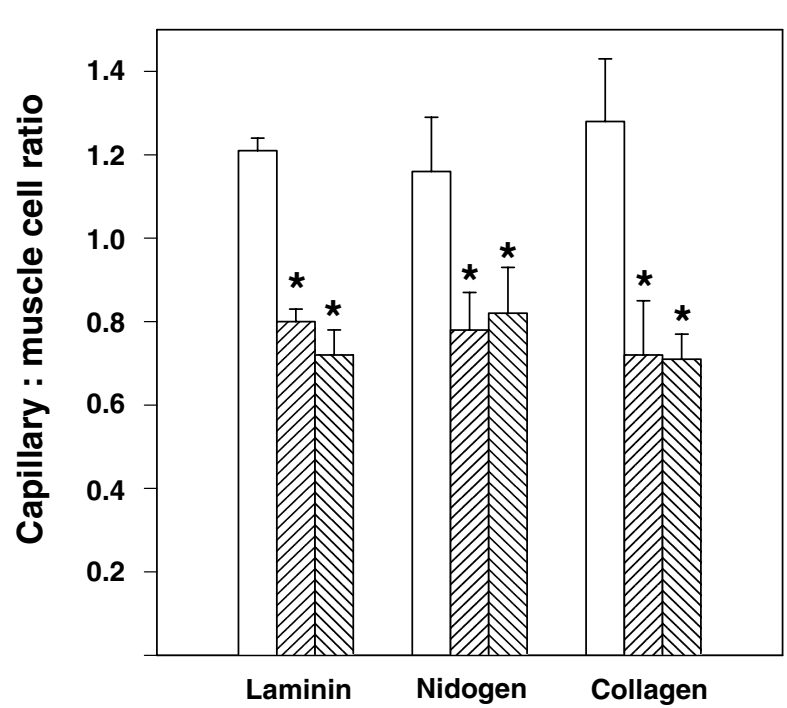

Fig. 5. Capillary:muscle cell ratios in mouse gastrocnemius cryosections immunostained with anti-BM antigens. Muscles were injected with either saline solution (open bars), $30 \mu \mathrm{g}$ BaPl (bars with diagonally ascending lines) or $5 \mu \mathrm{g}$ jararhagin (bars with diagonally descending lines). Tissue samples were collected $15 \mathrm{~min}$ after injections and immunostaining was performed as described in Materials and Methods. The total number of capillaries and muscle cells were counted in various sections, and the capillary:muscle cell ratio was calculated. Results are presented as mean $\pm \mathrm{SD}$ $(n=4) .{ }^{*} P<0.05$ when compared with capillary:muscle cell ratios of tissue from mice injected with saline solution. No significant differences $(P>0.05)$ were observed neither between samples of tissue injected with $\mathrm{BaP} 1$ and those injected with jararhagin nor between samples of a similar treatment immunostained for the three BM antigens.

sections (Fig. 5). The reduction in the immunostaining of $\mathrm{BM}$ markers in muscle fibers was not as drastic as that described for capillaries in severely affected areas, and it allowed the identification of contour of muscle fibers (Fig. 4). Nevertheless, muscle BM showed a decrease in the intensity of the fluorescence, and there was interruption in the continuity of the BM staining in some fibers (Fig. 4). These alterations in immunostaining for endothelial cells and $\mathrm{BM}$ proteins were evident as early as $15 \mathrm{~min}$ after $\mathrm{BaP} 1$ injection, and a similar pattern was observed at $1 \mathrm{~h}$. Hoechst staining revealed an increment in the amount of nuclei in $\mathrm{BaP} 1$-injected tissue, especially after $1 \mathrm{~h}$ of injection, but also at 15 min (not shown). This increment probably corresponds to leukocytes extravasated as a consequence of hemorrhage, and also to the initial inflammatory infiltrate arriving to damaged muscle. When $2 \mu \mathrm{g}$ of $\mathrm{BaP} 1$ were injected, no macroscopic hemorrhage developed in the muscle, and only a local edema was observed, with increase in the area of interstitial space. In these samples, the overall immunostaining for endothelial cell markers and for BM proteins was not reduced when compared with control samples (not shown). Thus, in conditions where hemorrhage did not occur, there was no evidence of loss of endothelial cell markers or capillary basement membrane antigens.

As previously described [27], injection of $5 \mu \mathrm{g}$ jararhagin induced a prominent hemorrhagic response in gastrocnemius muscle, which was of a roughly similar extent to that described above for $30 \mu \mathrm{g}$ BaP1. A similar immunocyto- chemical pattern as the one described after injection of $30 \mu \mathrm{g} \mathrm{BaP1}$ was observed, i.e. in areas of hemorrhage there was a loss in the staining for the endothelial cell markers and BM proteins in capillary vessels, whereas only a partial reduction in immunostaining to $\mathrm{BM}$ proteins was observed in skeletal muscle fibers in affected areas (Fig. 6). There was a significant drop in the capillary:muscle cell ratios in sections immunostained for the three BM antigens; such ratio did not differ from the ratio observed in sections of muscle affected by BaP1 (Fig. 5). Thus, the qualitative and quantitative alterations in the immunostaining of BM proteins laminin, type IV collagen and nidogen were similar when hemorrhagic doses of $\mathrm{BaP} 1$ and jararhagin were injected into muscle tissue. Moreover, the capillary:muscle cell ratio calculated for tissue sections immunostained for different BM antigens did not show any significant difference, either when comparing $\mathrm{BaP} 1$ and jararhagin or when comparing the three antigens among themselves $(P>0.05$; Fig. 5). In contrast, when these two SVMPs were compared on a similar molar basis, i.e. $5 \mu \mathrm{g}$ jararhagin and $2 \mu \mathrm{g} \mathrm{BaP1}$, the former induced hemorrhage and a drastic loss of immunostaining to endothelial cells and BM proteins, whereas the latter did not cause overt immunohistochemical alterations and no hemorrhage, in agreement with the higher hemorrhagic activity of jararhagin.

\section{Discussion}

Since the early descriptions of SVMPs and SVMPinduced hemorrhage, it has been postulated that a key step in the pathogenesis of microvascular damage is the enzymatic hydrolysis of $\mathrm{BM}$ components $[10,28]$. However, these observations were based primarily on in vitro degradation studies. In general terms, P-III SVMPs display a more potent hemorrhagic activity than P-I SVMPs. To explain this difference, three possibilities have been postulated: (a) P-III SVMPs may have a higher turnover rate on the hydrolysis of BM components; (b) different classes of SVMPs may have a different specificity concerning the substrates which are predominantly hydrolyzed, with P-III SVMPs having a higher activity on key BM components; and (c) the presence of disintegrin-like and cysteine-rich domains in P-III SVMPs may contribute to the targeting of SVMPs to relevant sites in the microvasculature $[3,5,7]$. This targeting is likely to depend on the presence of exosites to $\mathrm{BM}$ proteins or to the binding to endothelial cell integrins, thus positioning these enzymes at sites where they are able to degrade $\mathrm{BM}$ proteins and induce microvessel disruption more efficiently than P-I SVMPs, which lack these additional exosite-containing domains. The role of these additional domains has been recently supported by structural and functional observations $[19,29,30]$.

$\mathrm{BaP} 1$ and jararhagin showed a similar enzymatic activity, on a mass basis, on azocasein $(400 \mathrm{U} / \mathrm{mg}$ for jararhagin and $500 \mathrm{U} / \mathrm{mg}$ for $\mathrm{BaP} 1$ ). In contrast, the minimum hemorrhagic doses of these SVMPs are different, being $5 \mu \mathrm{g}$ for jararhagin [31] and $20 \mu \mathrm{g}$ for $\mathrm{BaP1}$ [20]; therefore, the 

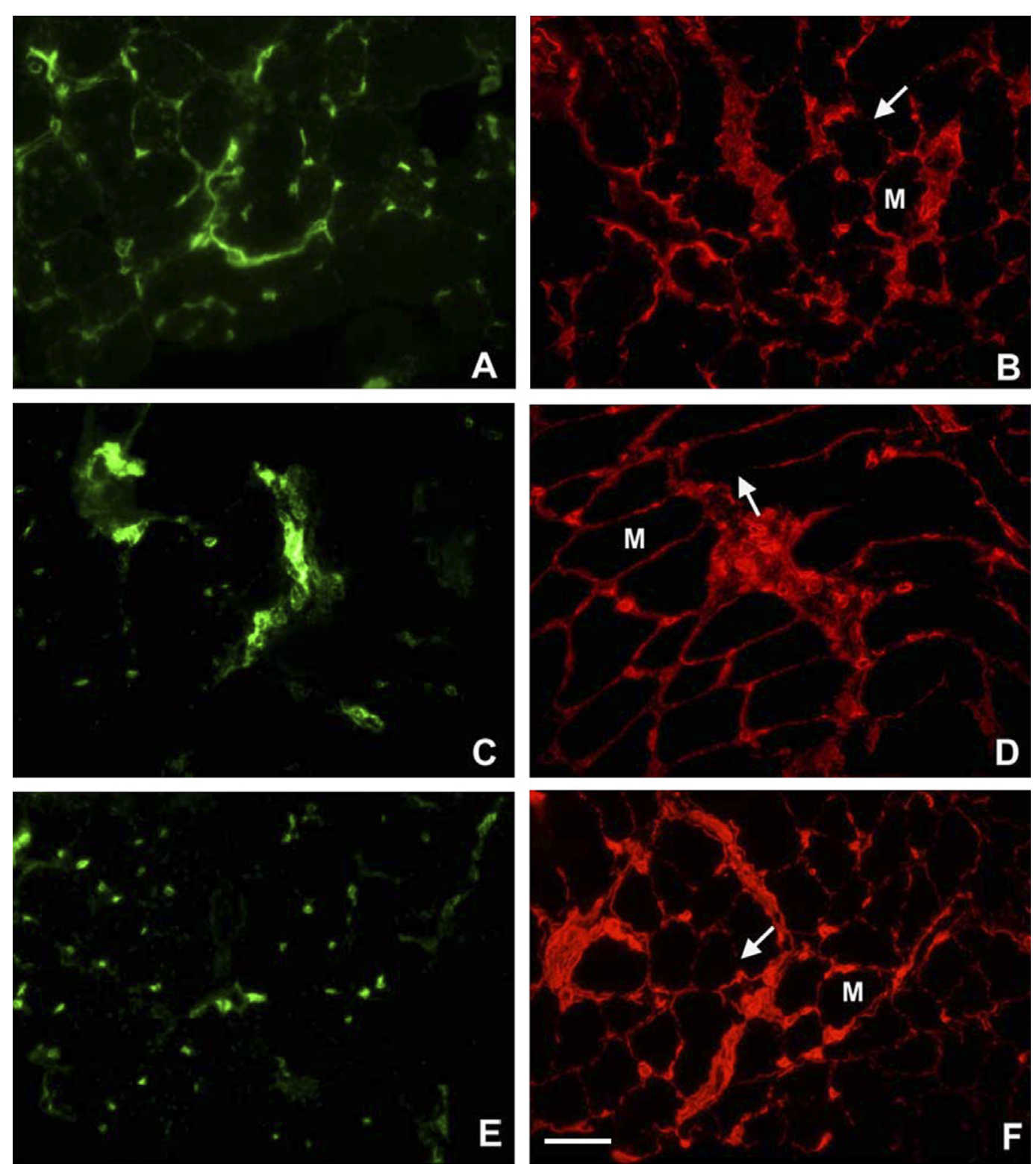

Fig. 6. Immunohistochemical staining for endothelial and BM markers in serial cryosections of skeletal muscle tissue (gastrocnemius) of mice 15 min after i.m. injection of $5 \mu \mathrm{g}$ jararhagin. Sections correspond to areas showing evidences of tissue damage, i.e. hemorrhage. The following primary antibodies were used: (A, C, E) anti-mouse vascular endothelial growth factor receptor 2 (VEGFR-2), (B) anti-mouse laminin, (D) anti-mouse nidogen, (F) anti-mouse type IV collagen. Sections A and B, C and D, and E and F correspond to serial sections from the same tissue sample. Immunostaining was performed as described in the legend of Fig. 3. There is a drastic reduction in the immunostaining of VEGFR-2 and BM markers in capillaries. There is a diffuse staining in (A) and (C) that does not correspond to endothelial cells. BMs of skeletal muscle cells show some alterations, i.e. reduction in the intensity of immunostaining and interruption in their continuity (arrow), although such alterations were less pronounced that those observed in capillaries. Bar represents $40 \mu \mathrm{m}$.

number of units of proteolytic activity in one minimum hemorrhagic dose corresponds to $2 \mathrm{U}$ for jararhagin and $10 \mathrm{U}$ for BaP1. Thus, the striking difference in hemorrhagic activity between these two proteinases cannot be explained on the basis of enzymatic turnover rate alone. Our present study also compared the patterns of degradation of Matrigel in vitro by these two SVMPs. The cleavage pattern on laminin and nidogen induced by $\mathrm{BaP} 1$ was similar to the one previously described for another class P-I SVMP, atrolysin $e$, from the venom of $C$. atrox [12]. On the other hand, jararhagin induced a somewhat different degradation pattern when compared with $\mathrm{BaP} 1$, especially regarding hydrolysis of laminin, which is slower and limited to the $\alpha$ chain. Jararhagin generates predominantly nidogen-derived fragments, which are similar to those described for the action of atrolysin $e$ [12].

The functional significance of the different patterns of BM degradation by hemorrhagic SVMPs is a relevant issue. BM plays a key role in the mechanical stability of the capillary wall, providing resistance to the distention of the capillary wall by the hemodynamic biophysical forces normally operating in the circulation [32,33]. BM is a 


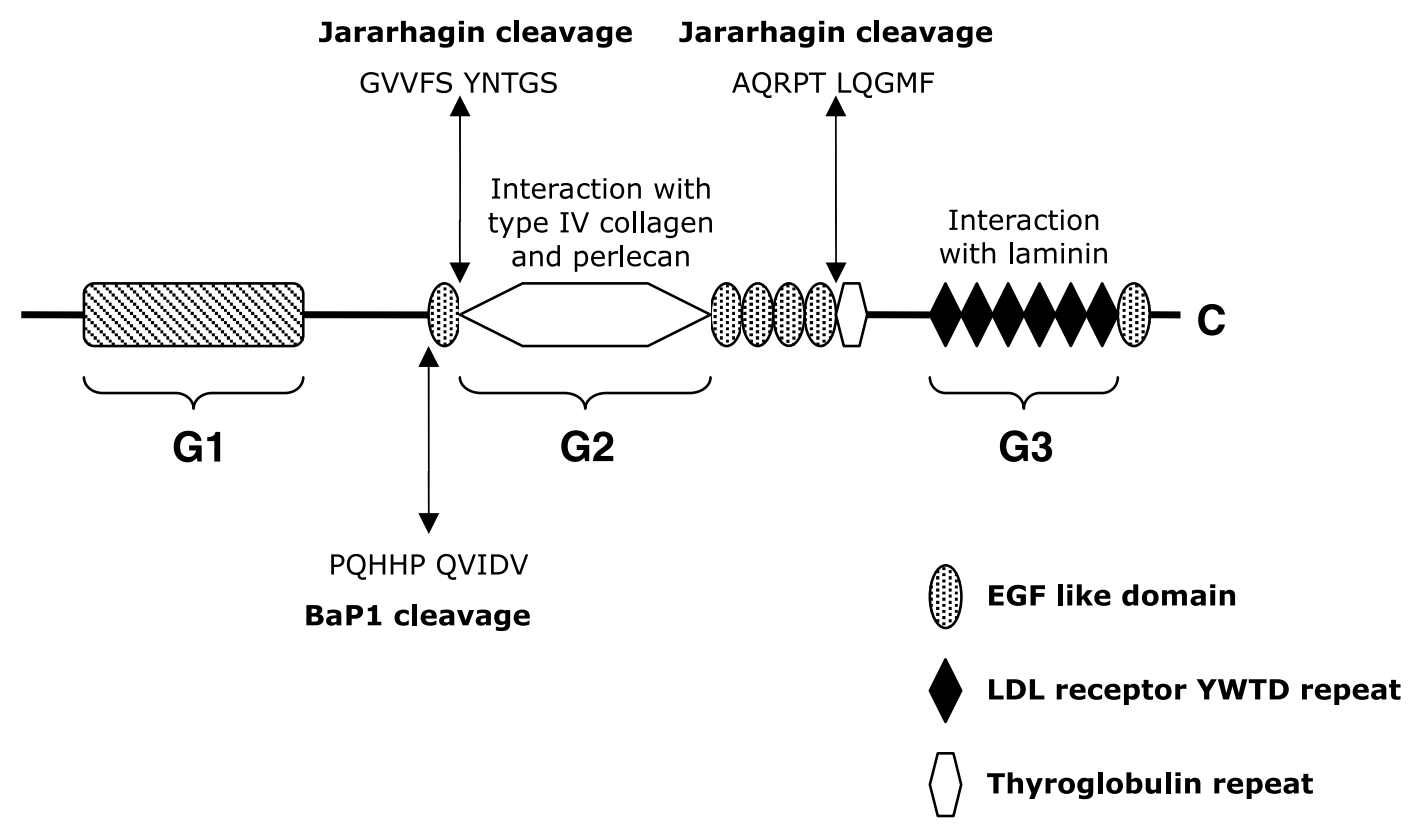

Fig. 7. Cleavage pattern of matrigel mouse nidogen in vitro by BaP1 and jararhagin. Cleavage sites were identified by N-terminal sequencing of the resultant fragments separated by SDS-PAGE. The regions in nidogen responsible for interaction with type IV collagen, perlecan and laminin are shown. The molecular organization of nidogen is based on [39], and the sequences of mouse nidogen were obtained from Swiss Prot data base (Accession No. P10493).

highly complex network of proteins, comprising predominantly laminin, type IV collagen and nidogen, together with the proteoglycan perlecan [16]. The observed differences in the cleavage sites of $\mathrm{BaP} 1$ and jararhagin on nidogen might be of functional relevance. Nidogen is a $150 \mathrm{kDa}$ protein comprising three globular domains which mediate the interaction with various BM components (Fig. 7) [34,35]. Domain G2 is involved in the interaction with type IV collagen and perlecan [35-37], whereas domain G3 mediates the interaction with laminin $\gamma$ chain $[35,36,38,39]$. Thus, nidogen plays a key role in the networking of type IV collagen and laminin in BMs. It is noteworthy that jararhagin cleaves nidogen at two sites, between Ser 351 and Tyr 352, and between Thr 839 and Leu 840, before the tyroglobulin-like repeat (Fig. 7). The last cleavage would provoke the separation between the molecular sites in nidogen that mediate the binding to type IV collagen and laminin, an event that is likely to promote a destabilization in the structure of nidogen, jeopardizing its role as an integrator of type IV collagen and laminin in BM (Fig. 7). In contrast, BaP1 cleaves nidogen between Pro 335 and Gln 336, just before the G2 domain; such cleavage would not separate the regions in nidogen responsible for interaction with type IV collagen and laminin. This difference in the cleavage of nidogen may contribute to the higher hemorrhagic activity of jararhagin.

Immunohistochemical experiments demonstrated that hemorrhagic SVMPs, at doses that induce hemorrhage, cause a drastic loss in the immunostaining of both endothelial cell markers and capillary BM components. In contrast, injection of $\mathrm{BaP} 1$ at a non-hemorrhagic dose did not provoke these alterations. This is the first immunohisto- chemical demonstration of in vivo BM degradation by snake venom hemorrhagic SVMPs. The loss of endothelial cell markers evidences a drastic endothelial pathology, in agreement with ultrastructural studies on the action of hemorrhagic SVMPs showing prominent alterations, leading to endothelial cell disruption, that occur within minutes of SVMP injection [40-42]. On the other hand, both qualitative and quantitative (i.e., capillary:muscle cell ratio) assessments revealed a conspicuous reduction in the immunostaining of BM antigens in capillaries upon treatment with hemorrhagic doses of these SVMPs. It is noteworthy that loss of immunostaining of BM antigens of capillaries in hemorrhagic areas was similar for the three BM antigens, thus suggesting an extensive loss in BM structure induced by these SVMPs in vivo. Interestingly, the pattern of limited proteolysis of BM proteins observed in vitro contrasts with the prominent loss of immunostaining of these proteins in affected capillaries in vivo. It is unlikely that such limited proteolytic profile would eliminate all the epitopes recognized by the polyclonal antibodies used in the immunohistochemistry experiments.

These apparently contradictory observations may be explained in the light of a hypothesis recently proposed to explain the mechanism of action of hemorrhagic SVMPs [7]. According to this model, capillary vessel disruption occurs in two stages: initially, there is limited proteolysis of BM proteins by SVMPs, as demonstrated in previous studies (see Ref. 5 for a review) and in our in vitro observations. As a consequence, the mechanical stability of BM is drastically weakened, and the hemodynamic biophysical forces normally operating in the circulation, i.e. wall tension and shear stress, provoke a distention in the capillary wall until its integrity is disrupted and extravasation 
occurs in an explosive fashion [7,42]. In the light of this hypothesis, it is suggested that such abrupt distention and rupture results in the loss of integrity not only of endothelial cells, but also of BM components, as observed at the ultrastructural level [41,42]. In agreement with this hypothesis, no evident ultrastructural changes occur in the $\mathrm{BM}$ of capillaries in tissue injected with BaP1 when blood flow was abrogated [42]. In these circumstances, it is likely that there is SVMP-induced limited proteolysis of BM components, but the lack of blood flow-dependent enhanced wall tension precludes the mechanical disruption of BM structure.

In contrast to the prominent reduction of $\mathrm{BM}$ immunostaining in capillaries where endothelial cells were damaged, the effects of hemorrhagic doses of $\mathrm{BaP} 1$ and jararhagin in the immunostaining of components of $\mathrm{BM}$ of skeletal muscle fibers occurred to a more limited extent. Despite subtle variations in the composition of $\mathrm{BM}$ in capillaries and skeletal muscle fibers [43], it is likely that SVMPs induce a similar pattern of limited proteolysis in their protein components. Nevertheless, the presence of hemodynamic mechanical forces promoting distention in capillaries, and the absence of similar forces in skeletal muscle, would explain why there is a widespread loss of BM immunostaining in affected capillaries whereas only a limited and partial loss occurs in BM surrounding muscle cells.

In conclusion, our observations indicate that, when injected at doses that provoke hemorrhage, both P-I and P-III SVMPs induce prominent loss of BM components in vivo, as judged by immunohistochemisty. Such drastic loss of capillary BM antigens in vivo may depend on both the limited enzymatic cleavage of BM components, and the disruption caused by biophysical forces operating in the microvasculature in vivo once BM stability has been debilitated. In vitro observations revealed a partially different pattern of limited proteolysis induced by these SVMPs on Matrigel. It remains to be determined whether such different hydrolytic patterns, as well as the higher hemorrhagic activity of jararhagin over BaP1, depend on differences in the metalloproteinase domain or on the ability of disintegrin-like and cysteine-rich domains of jararhagin to target it to specific sites in BM components of capillary blood vessels.

\section{Acknowledgments}

The authors thank Dr. Alexandra Rucavado and Javier Núñez (Instituto Clodomiro Picado), Dr. Ernesto Jiménez and Claudia Gutiérrez (Pathology Laboratory, Hospital San Juan de Dios, Costa Rica), Dr Kenneth S. Tung and Claudia Rival (Department of Pathology, University of Virginia), Dr Bojan Dragulev and Deju Wang (Department of Microbiology, Health Sciences Center, University of Virginia). This work was supported by Polifarma LTD (J.W.F.) and by Vicerrectoría de Investigación, Universidad de Costa Rica (Project 741-A4-061).
This work was carried out in partial fulfillment of the requirements for the Ph.D. degree for T. Escalante at the University of Costa Rica.

\section{References}

[1] D.A. Warrell, in: J. Meier, J. White (Eds.), Handbook of Clinical Toxicology of Animal Venoms and Poisons, CRC Press, Boca Raton, FL, 1995, pp. 493-594.

[2] D.A. Warrell, in: J.A. Campbell, W.W. Lamar (Eds.), The Venomous Reptiles of the Western Hemisphere, vol. II, Comstock, Ithaca, 2004, pp. 709-761.

[3] J.W. Fox, S.M.T. Serrano, Toxicon 45 (2005) 969-985.

[4] W. Bode, F.X. Gomis-Rüth, W. Stöckler, FEBS Lett. 331 (1993) $134-140$.

[5] J.B. Bjarnason, J.W. Fox, Pharm. Ther. 62 (1994) 325-372.

[6] J.M. Gutiérrez, A. Rucavado, Biochimie 82 (2000) 841-850.

[7] J.M. Gutiérrez, A. Rucavado, T. Escalante, C. Díaz, Toxicon 45 (2005) 997-1011.

[8] A. Ohsaka, in: C.Y. Lee (Ed.), Handbook of Experimental Pharmacology, vol. 52, Springer-Verlag, Berlin, 1979, pp. 480-546.

[9] J.B. Bjarnason, D. Hamilton, J.W. Fox, Biol. Chem. Hoppe-Seyler 369 (1988) 121-129.

[10] E.N. Baramova, J.D. Shannon, J.B. Bjarnason, J.W. Fox, Arch. Biochem. Biophys. 275 (1989) 63-71.

[11] E.N. Baramova, J.D. Shannon, J.B. Bjarnason, J.W. Fox, Matrix 10 (1990) 91-97.

[12] E.N. Baramova, J.D. Shannon, J.W. Fox, J.B. Bjarnason, Biomed. Biochim. Acta 4 (1991) 763-768.

[13] M. Maruyama, M. Sugiki, E. Yoshida, K. Shimaya, H. Mihara, Toxicon 30 (1992) 1387-1397.

[14] A. Rucavado, B. Lomonte, M. Ovadia, J.M. Gutiérrez, Exp. Mol. Pathol. 63 (1995) 186-199.

[15] J.B. Bjarnason, L.A. Hite, J.D. Shannon, S.J. Tapaninaho, J.W. Fox, Toxicon 31 (1993) 513.

[16] R. Kalluri, Nat. Rev. Cancer 3 (2003) 422-433.

[17] A.M. Moura-da-Silva, C. Marcinkiewicz, M. Marcinkiewicz, S. Niewiarowski, Thromb. Res. 102 (2001) 153-159.

[18] I. Tanjoni, D. Butera, L. Bento, M.S. Della-Casa, R. Marques-Porto, H.A. Takehara, J.M. Gutiérrez, I. Fernandes, A.M. Moura-da-Silva, Toxicon 42 (2003) 801-808.

[19] S.M.T. Serrano, L.G. Jia, D. Wang, J.D. Shannon, J.W. Fox, Biochem. J. 391 (2005) 69-76.

[20] J.M. Gutiérrez, M. Romero, C. Díaz, G. Borkow, M. Ovadia, Toxicon 33 (1995) 19-29.

[21] M.J.I. Paine, H.P. Desmond, R.D.G. Theakston, J.M. Crampton, J. Biol. Chem. 267 (1992) 22869-22876.

[22] L. Watanabe, J.D. Shannon, R.H. Valente, A. Rucavado, A. AlapeGirón, A.S. Kamiguti, R.D.G. Theakston, J.W. Fox, J.M. Gutiérrez, R.K. Arni, Protein Sci. 12 (2003) 2273-2281.

[23] W.J. Wang, C.H. Shih, T.F. Huang, Biochem. Biophys. Res. Comm 324 (2004) 224-230.

[24] U.K. Laemmli, Nature 227 (1970) 680-685.

[25] J.M. Gutiérrez, C.L. Ownby, G.V. Odell, Toxicon 22 (1984) 719-731.

[26] M.J. Plyley, A.C. Groom, Am. J. Physiol. 228 (1975) 1376-1383.

[27] P. Gallagher, Y. Bao, S.M.T. Serrano, G.D. Laing, R.D.G. Theakston, J.M. Gutiérrez, T. Escalante, P. Zigrini, A.M. Moura-da-Silva, R. Nischt, C. Mauch, C. Moskaluk, J.W. Fox, Arch. Biochem. Biophys. 441 (2005) 1-15.

[28] A. Ohsaka, M. Just, E. Habermann, Biochim. Biophys. Acta 323 (1973) 415-438.

[29] S. Takeda, T. Igarashi, H. Mori, S. Araki, EMBO J. 25 (2006) 2388-2396.

[30] A.S. Kamiguti, P. Gallagher, C. Marcinkiewicz, R.D.G. Theakston, M. Zuzel, J.W. Fox, FEBS Lett. 549 (2003) 129-134.

[31] G.D. Laing, P.B. Clissa, R.D. Theakston, A.M. Moura-da-Silva, M.J. Taylor, Eur. J. Immunol. 33 (2003) 3458-3463. 
[32] J. Lee, G.W. Schmid-Schönbein, Ann. Biomed. Engin. 23 (1995) 226-246.

[33] B.J. Ballerman, A. Dardik, E. Eng, A. Liu, Kidney Int. 54 (1998) S100-S108.

[34] M. Dziadek, M. Paulsson, R. Timpl, EMBO J. 4 (1985) 2513-2518.

[35] J.W. Fox, U. Mayer, R. Nischt, M. Aumailley, D. Reinhardt, H. Wiedermann, K. Mann, R. Timpl, T. Krieg, J. Engel, M.L. Chu, EMBO J. 10 (1991) 3137-3146.

[36] M. Aumailley, C. Battaglia, U. Mayer, D. Reinhardt, R. Nischt, R. Timpl, J.W. Fox, Kidney Int. 43 (1993) 7-12.

[37] M. Kvansakul, M. Hopf, A. Ries, R. Timpl, E. Hohenester, EMBO J. 19 (2001) 5342-5346.
[38] U. Mayer, E. Kihfeldt, R. Timpl, Ann. NY Acad. Sci. 857 (1998) 130-142.

[39] J. Takagi, Y. Yang, J.H. Liu, J.H. Wang, T.A. Springer, Nature 424 (2003) 969-974.

[40] C.L. Ownby, J.B. Bjarnason, A.T. Tu, Am. J. Pathol. 93 (1978) 201-218.

[41] L. Moreira, G. Borkow, M. Ovadia, J.M. Gutiérrez, Toxicon 32 (1994) 977-987.

[42] J.M. Gutiérrez, J. Núñez, T. Escalante, A. Rucavado, Microv. Res. 71 (2006) 55-63.

[43] R. Hallmann, N. Horn, M. Selg, O. Wendler, F. Pausch, L.M. Sorokin, Physiol. Rev. 85 (2005) 979-1000. 